\title{
Pharmacotherapy virtual attachment during COVID-19 pandemic: Use of online experiential assessment in a Malaysian pharmacy school
}

\author{
M.H. Elnaem*, N.I.M. Nazar, N.S.A. Rahman \\ Department of Pharmacy Practice, Kulliyyah of Pharmacy, International Islamic University Malaysia, Malaysia
}

\author{
Keywords \\ Experiential Learning \\ Pharmacotherapy \\ Malaysia \\ Online Teaching \\ Virtual OSCE
}

\section{${ }^{*}$ Corresponding author: drmelnaem@iium.edu.my}

\begin{abstract}
Summary: This case study aims to report the efforts made by International Islamic University Malaysia to pursue the experiential pharmacotherapy attachment for the final-year of pharmacy students via online mediums in the light of the COVID-19 restrictions on face-to-face clinical experiential learning. A four-week virtual pharmacotherapy experiential attachments were redesigned and conducted via an online platform (Google Meet). Students were required to have a two-hour virtual discussion and consultation session with their preceptors twice a week throughout the attachment period. In each week, students were provided with real-life clinical cases that were chosen by their preceptors from the Department of Pharmacy Practice's clinical case repository. Students were assessed via clinical case reports, virtual seminar presentations, weekly virtual discussions with preceptors, and virtual objective structured clinical examinations. All the virtual sessions were conducted on a synchronous basis to assure students' active participation. Alongside this, a revised online final assessment was also designed.
\end{abstract}

\section{Background and Context}

Pharmacotherapy experiential learning offered for final-year pharmacy students was an excellent platform to consolidate the class-based teaching and to integrate it with real clinical scenarios. With the restrictions imposed on all higher learning institutions and teaching hospitals amid the COVID-19 pandemic, the Department of Pharmacy Practice were forced to redesign their approach to proceed with the students' clinical attachments using online activities (Hodges et al., 2020). The simulated cases acted as the basis for the approach to proceed with the attachments without compromising the quality of this practice-based learning experience for the students. With the support of International Islamic University Malaysia's academic management, flexibility was given for the educators to do the required revisions to the content and assessments of their courses, particularly the experiential courses to be delivered remotely (Lederman, 2020). The innovations were needed to accommodate the desperate need to provide more interaction with educators, simulated scenarios, and practice-based assignments. The innovations were also required to proceed with the conventional experiential assessment, e.g. the objective structured clinical examinations (OSCEs) on a virtual platform without face-to-face physical interactions with students (Lucas \& Forrest, 2020). In this case study, the authors discuss the innovations in the design and assessment of the pharmacotherapy virtual experiential component undergone due to COVID-19 restrictions.

\section{Educational Description}

The final-year pharmacotherapy experiential module was originally designed as a four-week placement in hospital settings on a rotational basis. The delivery was wholly 
dependent on the students' interactions in real-life scenarios under the supervision of registered practitioners in the practice setting. Due to the COVID-19 pandemic and its related restrictions on all sorts of fieldwork teaching and learning, the course had to be re-designed. This course was designed initially to expose students to hospital pharmacy services and clinical pharmacy in real practice settings over a four-week period. In responding to the COVID-19 pandemic, the duration was shortened to three weeks. There were a total of 114 students and 12 academic staff involved in the re-design of the learning and assessment activities of this course.

Virtual clinical case discussions were conducted twice weekly and were dedicated to clinical experiences based on real hospital cases from the repository of clinical cases in the Department. The students were assigned their cases through Google Classroom; students could then discuss issues with their clinical preceptor through Google Meet. A total of four cases were allocated to every student over the two weeks, and students were asked to write clinical case reports for each one which were assessed by different clinical preceptors. On a rotational basis, every academic staff member was responsible for two or three students for the virtual clinical discussions to make sure they were able to cover all aspects of the cases with their students comprehensively. In previous years, every staff member used to receive double this number.

Alongside the care reports, every student was asked to choose one case to present in a seminar presentation in front of their colleagues and clinical preceptors. The presentations were conducted online through Google Meet and were assessed by the assigned faculty staff for each group. The weightage of all online discussions, report submissions and presentations was $50 \%$ of the overall assessment. The remaining $50 \%$ was distributed between online OSCEs (20\%) and final assessment (30\%).

A written protocol outlining all the procedures and steps to be followed was developed as a preparation for the virtual OSCEs. Then, briefing sessions were conducted for both students and examiners, separately. After that, trial run sessions (mock OSCE) were conducted online through the same platform as the main OSCE (Google Meet) to make sure that both students and examiners are fully prepared to proceed to the main OSCE. The main OSCE was conducted as synchronous sessions; it involved 12 examiners and ten rounds. A total of ten different cases of comparable difficulty were offered to students. This was followed by a final assessment, delivered online through Google Classroom; the exam duration lasted longer due to the need to address online examination challenges such as the time required to switch between different questions (staff structured the online evaluation as one question per page). The initial feedback showed the acceptability of the redesigned virtual attachment with some reported challenges from students (failing internet connections) and faculty (increased workload) perspectives.

\section{Outcomes and Recommendations}

The main objective of the case study was to provide a reasonable solution for graduating students to pursue a quality-driven simulated pharmacotherapy virtual attachment amid the COVID-19 restrictions. In view of the unforeseen limitations of the pandemic and the support and training provided by academic management, it was time to redesign the final-year pharmacotherapy experiential attachment as a virtual experience that has value and input from real clinical settings. Redesigning the programme was an eye-opening experience, which showed how the Department can further integrate online tools and platforms into students' studies to enrich, not to replace, the clinical placement. The Department of Pharmacy Practice at the International Islamic University Malaysia is now more able to proceed with the integration of online learning into the experiential components, not just the emergency remote teaching.

Beside achieving the desired learning outcomes, the insights from both students and faculty members were fundamental in the overall evaluation of the virtual clinical experiential attachment. Overall, the new design and assessment were successfully received by the students although a few challenges arose about maintaining students' active participation during the live sessions and technical issues of internet connection stability. From the faculty's perspective, the experience was overall well-conducted despite the challenge of having to quickly adapt to a new style of teaching. However, there was an increasing trend in the number and duration of online meetings coupled with several new requirements of documentations and briefing sessions for both students and preceptors that posed new challenges for staff.

Developing a way to successfully integrate online tools into the course will be essential in the future to add value to experiential learning without additional burden on the faculty staff.

\section{References}

Hodges, C., Moore, S., Lockee, B., \& Trust, T. (2020) The Difference Between Emergency Remote Teaching and Online Learning. Educause Review. Available at: https://er.educause.edu/articles/ 2020/3/the-difference-between-emergency-remote-teaching-andonline-learning

Lederman, D. (2020) Will Shift to Remote Teaching Be Boon or Bane for Online Learning? Inside Higher Ed. Available at: https:// www.insidehighered.com/digital-learning/article/2020/03/18/mostteaching-going-remote-will-help-or-hurt-online-learning

Lucas, C., \& Forrest, G. (2020) Virtual OSCEs - Challenges and Considerations for Pharmacy Education? Pulses: Promoting scholarly dialogue in pharmacy education. Available at: https:// cptlpulses.com/2020/06/18/virtual-osces/ 